\title{
Experimental studies of a single flexibly-mounted rod in a triangular rod bun- dle in cross-flow
}

\author{
Sabine Upnere ${ }^{1,2, \star}$, Normunds Jekabsons ${ }^{3,2}$, Sergejs Dementjevs ${ }^{4}$, and Michael Wohlmuther ${ }^{4}$ \\ ${ }^{1}$ Institute of Mechanics, Riga Technical University, Latvia \\ ${ }^{2}$ Ventspils University College, Latvia \\ ${ }^{3}$ Faculty of Physics and Mathematics, University of Latvia, Latvia \\ ${ }^{4}$ Paul Scherrer Institute, Switzerland
}

\begin{abstract}
Experiments on flow-induced vibrations using a closely-packed triangular rod array with a pitch-todiameter ratio of 1.1 in water cross-flow was carried out at Paul Scherrer Institute. The bundle consists of 21 row of five rods in each one. Single flexibly-mounted test rod (TR) is in the fourth row in an otherwise fixed array. The test rod can freely move in the transverse and in-line direction. Two accelerometer sensors were attached at both ends of the TR to measure the rod response on the fluid flow. The effect of flow rate on the stability of the flexibly-mounted TR has been analysed. During experiments, it reveals a set of conditions and tendencies for the flow-induced vibration in the closely-packed multi-rod system.
\end{abstract}

In mechanical, civil or nuclear engineering communities for the structural health monitoring vibration analysis is commonly used. This work is a contribution to investigations directed on development of SINQ (Swiss Spallation Neutron Source) target accident protection system. SINQ target is located in Paul Scherrer Institute (PSI).

The mock-up of SINQ target with single flexiblymounted test rod (TR) in an otherwise rigid triangular rod array, with a pitch-to-diameter ratio of 1.1 has been used to experimentally study the flow-induced vibrations (FIV) due to the water cross-flow (flow is perpendicular to the rod axis). Cross-flow-induced vibrations are responsible for the vast majority of failures [1] in the multi-tube systems. Due to the mounting of the TR, the oscillating system has relatively small stiffness features.

Although an experimental investigation of the system could be expensive compared with analytical or numerical solutions, it is necessary to get an exact knowledge of the excitation mechanism of FIV in the particular rod array. The behaviour of the vibrations in the rod bundle is tightly related to the geometrical or mechanical variation of the array layout [2]. In the case of liquid as a surrounding substance, there are several basic principles of FIV excitation, for example, vortex shedding, turbulence buffeting, fluid-elastic instability [3], [4]. Weaver and Grover [5], [6] experimentally found all mentioned excitation mechanisms in a tube bank with 19 flexibly mounted tubes surrounded by 116 rigid. Fluid excitation forces originated from vortex shedding and turbulence are experimentally measured in tightly packed rigid tube bundle by Liu et al. [7]. They conclude that a rod position is the most critical

\footnotetext{
^e-mail: upnere@gmail.com
}

point regarding excitation forces acting on the system. Lin and $\mathrm{Yu}[8]$ have done experiments to analyse the influence of surrounding cylinders on critical velocity or vibration amplitude. Experimental results showed that the cylinder induced by the cross-flow vibrates initially only at its natural frequency around the onset of fluid-elastic instability but increasing the fluid velocity the cylinder vibrations include the frequency components of the surrounding cylinders too.

In the present study, non-contact measurements are used to analyse the TR response to environmental changes. Experiments were done at PSI. The amplitudes, oscillation orbits and frequency spectra of the TR vibration are obtained by measurements of accelerometers and laser sensors. A set of conditions and tendencies for the FIV in the very closely-packed multi-rod system is revealed using experimental results.

\section{Experimental set-up}

The experimental installation was developed at PSI. It consists of rectangular water channel with 21 row of rigid, cylindrical stainless steel rods. Rods are rigidly fixed in hollows in the plexiglass cover. All rods are identical, 30 $\mathrm{mm}$ long with $8 \mathrm{~mm}$ diameter. Each row contains five rods or four rods and two half-rods, see figure 1 . The rods are arranged in a normal triangular array with a small pitchto-diameter ratio $(P / d=1.1)$.

Based on the conclusion by Weaver and El-Kashlan [9] that the critical tubes for instability are in the third and fourth rows the single flexibly-mounted test rod (TR) is located in the middle of the fourth row. Two cylindrical elastic beams allow free movement of the TR in the transverse 


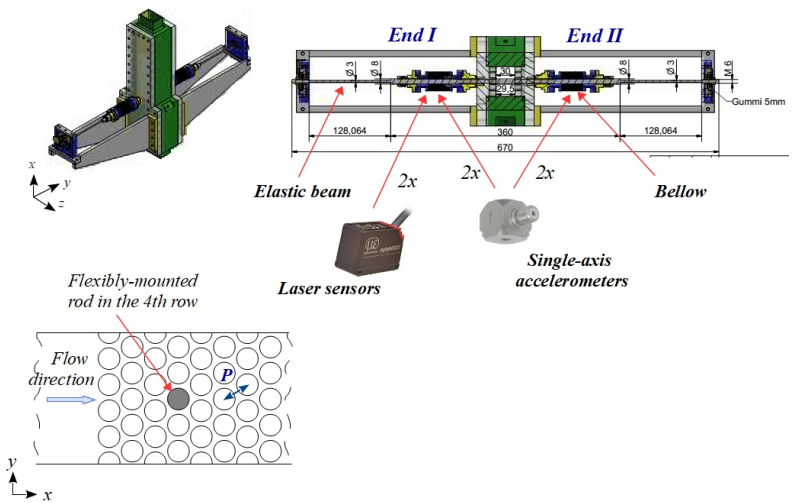

Figure 1. Experimental set-up

and in-line direction. Maximum oscillation amplitude is 1 $\mathrm{mm}$. Bellows at the rod ends provide waterproofing.

Maximum flow rate, $Q_{\max }$, in the channel inlet is $2.9 \mathrm{l} / \mathrm{s}$ or $2.06 \mathrm{~m} / \mathrm{s}$. The Reynolds number based on the maximum inlet velocity $U_{\infty}$ and cylinder diameter $d$ is 16454 . The mean gap flow velocity (1) at maximum inlet velocity corresponds to Reynolds number of 181280 . Therefore in the water flow in the rod bundle is fully developed turbulence.

$$
U_{p}=\frac{U_{\infty} P / d}{P / d-1}
$$

The coordinate system (Cartesian) is defined such that the $\mathrm{x}$-axis points in the direction of the flow, the $\mathrm{y}$-axis in the perpendicular direction and the $\mathrm{z}$-axis is the axis of the rod and is pointing out of the plane in the longitudinal direction of the rod. The origin of the coordinate system is in the centre of the oscillating rod.

\subsection{Sensors}

Non-contact measurements have been done. The instrumentation includes four single-axis accelerometers and two laser triangulation sensors.

Kistler's 8640A5 accelerometers with sensitivity 1000 $\mathrm{mV} / \mathrm{g}$ and frequency response of $0.5-3000 \mathrm{~Hz}$ are used for acceleration measurements. On the TR End I has been applied two sensors, one for perpendicular (Acc1) and one for parallel (Acc2) direction concerning to the flow. In the same manner, the second end (End II) of the TR is instrumented with two accelerometers Acc3 and Acc4.

Displacements of the flexibly-mounted TR are measured using optoNCDT 1320 laser triangulation sensor with $1 \mu \mathrm{m}$ repeatability. Two laser sensors are installed on the TR End I, one parallel (L1) and one perpendicular (L2) to the flow. Locations of the sensors can see in the figure 1.

Measurements show that analysed system has a high level of environmental noise and used sensors are not quite sensitive in cases when the flow rate is low $(Q<1 \mathrm{l} / \mathrm{s})$, see figure 2.

Due to the laser sensor lower accuracy, if compared with an accelerometer in given system, the analysis mostly is based on TR acceleration measurements.
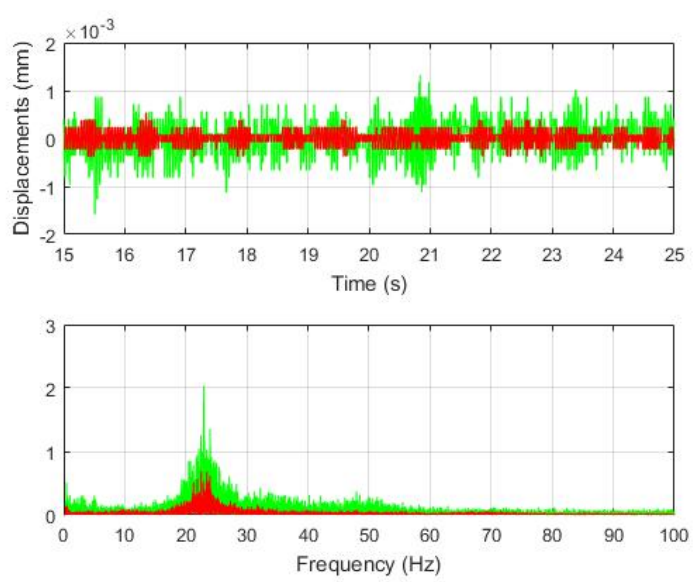

Figure 2. Measured displacements in $\mathrm{mm}$ when flow rate $Q=0$ 1/s, red line and $Q=0.53 \mathrm{l} / \mathrm{s}$, green line

\section{Mathematical formulations}

To obtain displacements from the accelerometer time history the following algorithm are used:

- $\ddot{X}(\omega)=F F T(\ddot{x}(t))$

- $\dot{X}(\omega)=\frac{\ddot{X}(\omega)}{\omega i}$

- $X(\omega)=-\frac{\ddot{X}(\omega)}{\omega^{2}}$

- $\dot{x}(t)=\operatorname{IFFT}(\dot{X}(\omega))$

- $x(t)=\operatorname{IFFT}(X(\omega))$

$\ddot{x}$ is measured acceleration, FFT is Discrete Fourier transform, IFFT is Inverse Fourier transform, and $\omega$ is a frequency. Validation of the applied algorithm is done using measurements of laser sensors.

The Power Spectral Density (PSD) where the power is normalized by the frequency resolution is used in the frequency domain analysis.

$$
P S D=\frac{1}{F_{S} \cdot N}|X(\omega)|^{2},
$$

where $F_{s}$ is sampling frequency and $N$ is a number of samples.

For displacement calculations and spectral analysis of the behaviour of the TR in cross-flow, the MATLAB scripts have been developed.

\subsection{Comparison of transitional and rotational movement}

For modelling and simulation purposes it would be useful to reduce the complexity of the analysed oscillation system. One way would be to move from 3-dimensional to 2-dimensional cases. To check if this is acceptable in a given case, transitional and rotational movements of the TR are compared. The mass-damper-spring system (see figure 3) has been used for evaluation.

Lagrangian equations (3) have been drawn up for two generalised coordinates: a displacement of the TR midpoint $x$ and angle $\theta$ of rotation around the centre of inertia. 


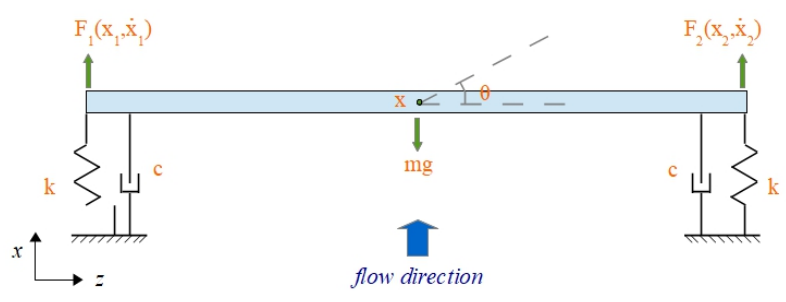

Figure 3. Mass-damper-spring system

Both generalized coordinates are used for perpendicular and parallel axis.

$$
\frac{d}{d t}\left(\frac{\partial L}{\partial \dot{q}_{i}}\right)-\frac{\partial L}{\partial q_{i}}=F_{i}, \quad i=1,2,3,4,
$$

where $L=T-V, T$ is kinetic energy, $V$ is potential energy, $q_{i}$ is generalized coordinates and $F_{i}$ is generalized forces.

Since it is not possible to compare coordinate and angle in a direct way, the kinetic energy is used. The ratio of kinetic energies of the transitional and rotational movements (4) are shown in figure 4.

$$
\frac{T_{x}}{T_{\theta}}=\frac{1 / 2 m \dot{x}^{2}}{1 / 2 I \dot{\theta}^{2}}
$$

where $m$ is mass and $I=m L^{2} / 12$.

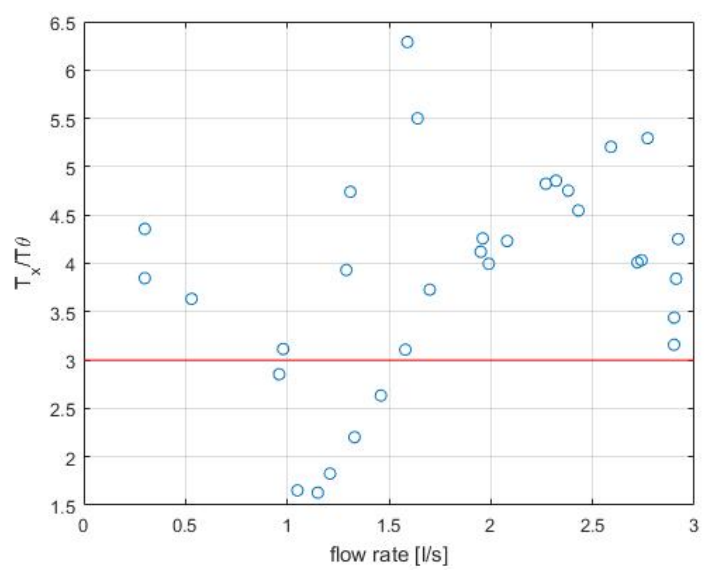

Figure 4. Ratio of the kinetic energies of $x$ and $\theta$

As can see from figure 4 , in most cases the kinetic energy of the displacement of the midpoint is at least 3 times larger than the kinetic energy of the rotation, therefore, can assume that in these cases the $2 \mathrm{D}$ approximations are permissible.

\section{Experimental results}

To find sensitive or hard-to-calculate system constants for the numerical and analytical models the force hammer tests are done. Different flow rates have been used to analyse the relationship between the flow and oscillation frequencies as well between flow and oscillation amplitudes.

\subsection{Error estimation}

Random features are observed in virtually all measurements [10]. To estimate random error, the standard deviation $\sigma$ of acceleration and displacement measurements at maximum flow rate has been calculated. Average standard deviation and standard error (5) of four experiments are summarized in table 1. Measurements of the rod End I response is chosen (Acc1, Acc2 and L1, L2 sensors).

$$
e_{\sigma}=\frac{\sigma}{\sqrt{n_{\text {exp }}}}
$$

where $e_{\sigma}$ is a standard error, $n_{\text {exp }}$ is the number of experiments.

Table 1. Standard deviation and error of measurements of Acc1, Acc2, L1 and L2 sensors

\begin{tabular}{rrrrr}
\hline & Acc1 & Acc2 & L1 & L2 \\
\hline$\sigma$ & 0.1984 & 0.2014 & 0.0089 & 0.0079 \\
$e_{\sigma}$ & 0.0992 & 0.1007 & 0.0047 & 0.0037 \\
\hline
\end{tabular}

From table 1 follows that accelerometer measurements are more dissipated. Therefore laser sensors have the smaller standard error comparing to accelerometers.

\subsection{Damping ratio of the system}

The maximum amplitudes of the TR oscillations during the hammer tests correspond to the amplitude when the flow rate is higher than $2.5 \mathrm{l} / \mathrm{s}$. The system damping ratio is found using a logarithmic decrement. The logarithmic decrement is calculated by the following equation:

$$
\lambda=\frac{1}{N} \ln \frac{x(t)}{x(t+N T)},
$$

where $\lambda$ is a logarithmic decrement, $N$ is the integer of successive, positive peaks, $x(t)$ is the amplitude at time $t$, $T$ is the oscillation period. The damping ratio, $\zeta_{x}=0.020$ and $\zeta_{y}=0.019$ with standard deviations $\sigma_{x}=0.003$ and $\sigma_{y}=0.0017$, respectively, has been obtained.

The impulse of the unity force is used as excitation in hammer test. The transfer function $H$ from force $F$ to acceleration $a$ is:

$$
H(s)=\frac{a(s)}{F(s)}=\frac{1}{m} \frac{s^{2}}{s^{2}+2 \zeta \omega_{n} s+\omega_{n}^{2}},
$$

where $\omega_{n}=\sqrt{k / m}$ and $s$ is Laplace operator. Example of Bode diagram with frequency response and phase shift is shown in figure 5.

\subsection{The influence of the rod mass}

The damage of the rod may result in a reduction in the mass of the rod. To check is the rod mass reduction observable in the frequency or space domain in the experimental installation is attached two mass elements at the TR ends increasing the total TR mass, see figure 6.

The larger mass of the TR causes oscillation amplitude increase and frequency decrease. In the figure 7 is shown 


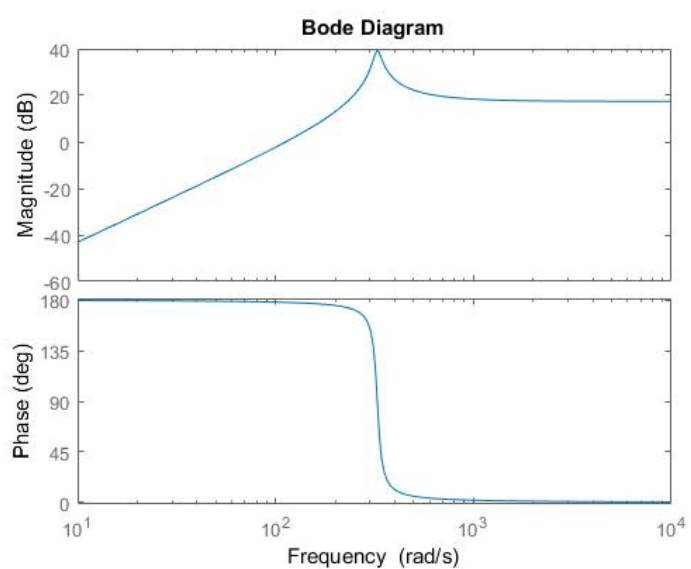

Figure 5. Bode diagram

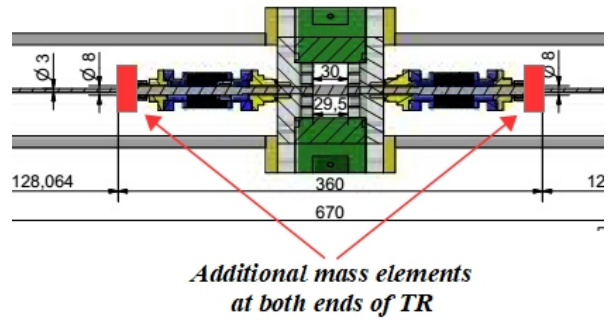

Figure 6. Experimental installation with increased mass of the TR

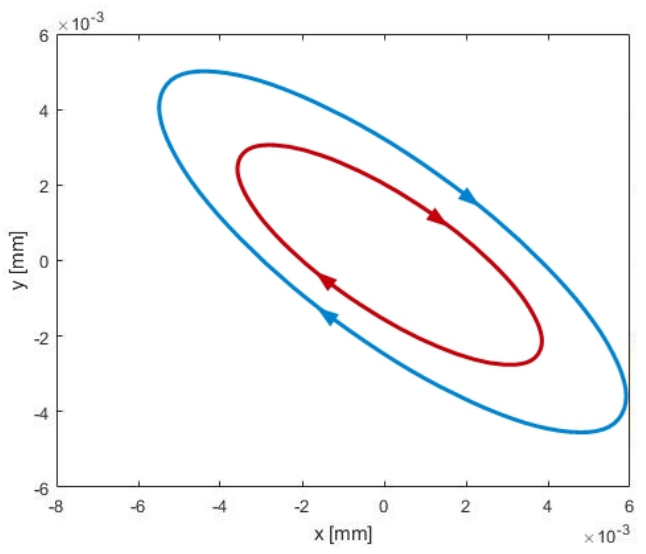

Figure 7. Left: TR movement in $\mathrm{x}-\mathrm{y}$ plane $(\mathrm{mm}) ; m_{0}$ - blue line, $m_{1}$ - red line.

TR movement in the $\mathrm{x}-\mathrm{y}$ plane with original mass, $m_{0}=$ $469 \mathrm{~g}$ (red line) and with larger mass, $m_{1}=581 \mathrm{~g}$ of the rod (blue line).

Measurements show that increasing the mass by 1.24 times causes frequency decrease by 1.13 times. The original mass gives the stiffness coefficient $k$ of $44283 \mathrm{~N} / \mathrm{m}$. Using calculated stiffness $k$ from the original case the frequency ratio is $1 \%$ larger. It can conclude that the fluid added mass has the minor influence in a particular case.
The TR mass changes are detectable from the hammer test in both in the frequency spectra (especially in parallel direction) and in the space domain.

\subsection{Maximum frequencies depending on flow rate}

PSD is used to analyse obtained accelerometer data in the frequency domain. In figure 8 is shown three maximum frequencies of each accelerometer measured in one experimental case.

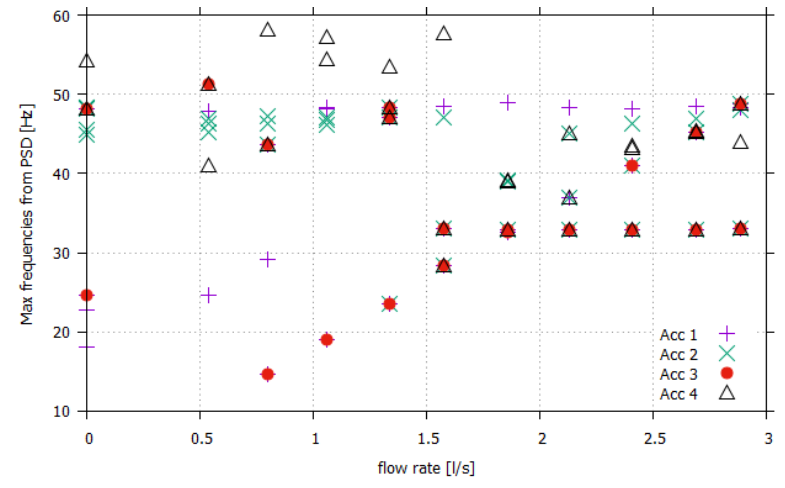

Figure 8. Maximum frequencies $(\mathrm{Hz})$ from the accelerometer measurements

In general, all registered frequencies can divide into three groups: a) frequencies around $50 \mathrm{~Hz}$; b) frequencies around $32 \mathrm{~Hz}$ starting after $1.5 \mathrm{l} / \mathrm{s}$; c) gradually growing frequencies when a flow rate is higher than $0.8 \mathrm{l} / \mathrm{s}$.

First two groups are related to the system and environmental noises. The last one should describe the characteristic frequency of the TR due to the flow. The PSD spectra for large flow rates is given in figure 9. As mentioned by Ziada [11], a narrow band peak indicates that it is basically a periodic phenomenon. As illustrated in figure 9, the linear relationship between the frequency, $f$ and the flow rate, $Q$ has been observed.

\subsection{Oscillation mechanisms}

The probability $P_{i},(8)$ is used to find areas in the x-y plane where the end of the TR is the most common during the time. This allows to better understand causes of the rod excitation.

$$
P_{i}=\frac{n_{i}(x)}{N}
$$

where $n_{i}(x)$ is number of samples in given area $i, N$ is number of total samples.

The interesting part of the $\mathrm{x}-\mathrm{y}$ plane is split into small squares and for each of these areas $P_{i}$ is calculated, see figure 10.

An analyse of the measured TR movement in the $\mathrm{x}$ $y$ plane depending on the water flow velocity shows two possible oscillation scenarios. In one case, the turbulent fluctuations are dominated by the system. If the behaviour of the oscillation system is dictated by the turbulence, with a higher probability the rod will be around the zero point, see figure 11 . In this case, cannot detect changes of the 


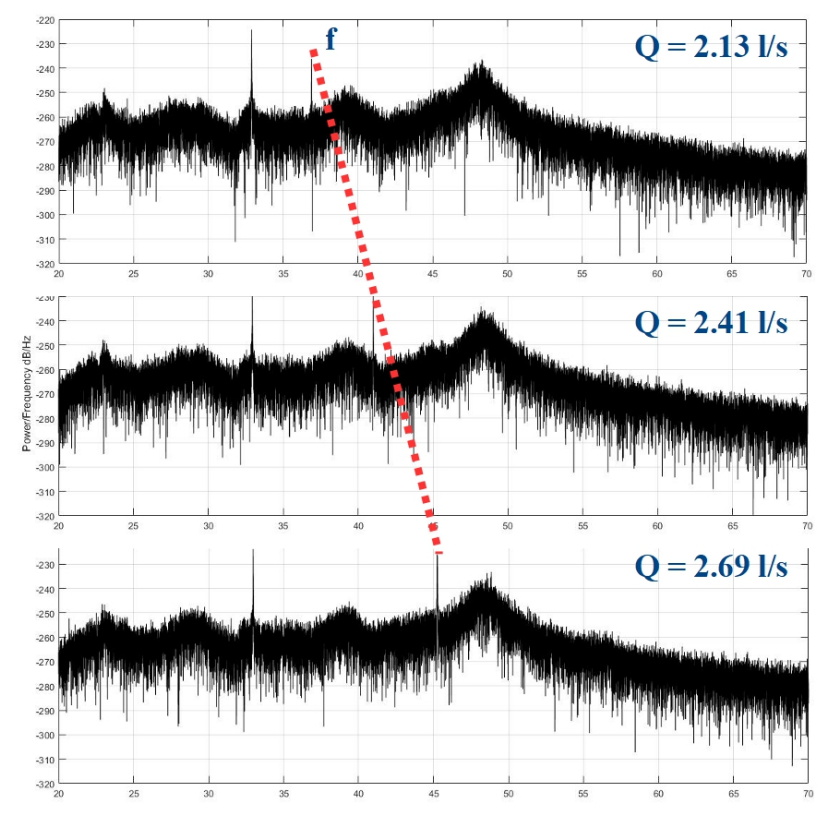

Figure 9. Typical spectra of PSD. With dotted line is shown a frequency, $f$ depending on the flow rate, $Q$. On x-axis is frequencies in $\mathrm{Hz}$ and on $\mathrm{y}$-axis is power/frequency in $\mathrm{dB} / \mathrm{Hz}$

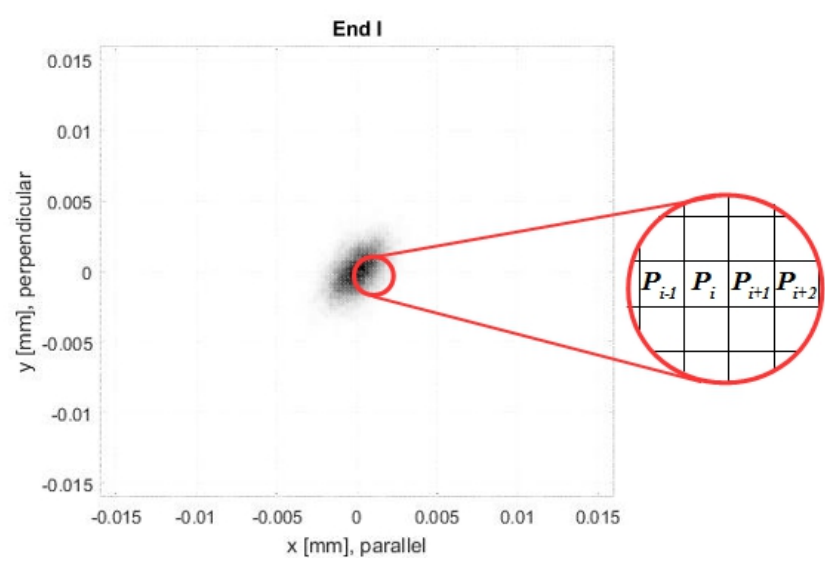

Figure 10. The plane splitting for the calculations of the probability function

characteristic frequencies of the system. The TR response on fluid flow is random and no periodic motion has been observed.

When in the probability graph is observed two maximum points, the rod has two unstable equilibrium states and its oscillation behaviour can describe as the pendulum system (see figure 12). In this case, can analyse processes related with theses system frequencies.

The boundary when the analysed system starts work as a pendulum system can be found investigating TR oscillation amplitude depending on the flow rate. Figure 13 shows that after $2.2 \mathrm{l} / \mathrm{s}$ the vibration amplitude of the TR starts to increase. When the flow rate is between 2.7 and $2.8 \mathrm{l} / \mathrm{s}$, the oscillation amplitude reaches its maximum. After maximum, the TR amplitude decrease although the flow velocity continues to grow. Thus, at high flow rates,

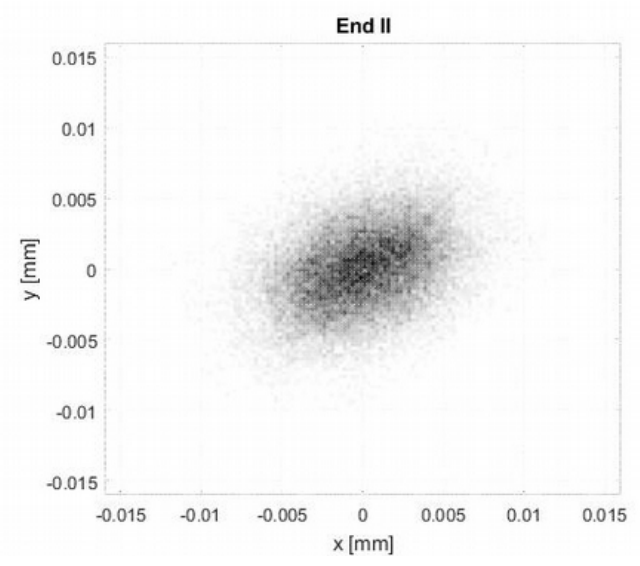

Figure 11. Probability of the TR location if turbulent fluctuations are dominant

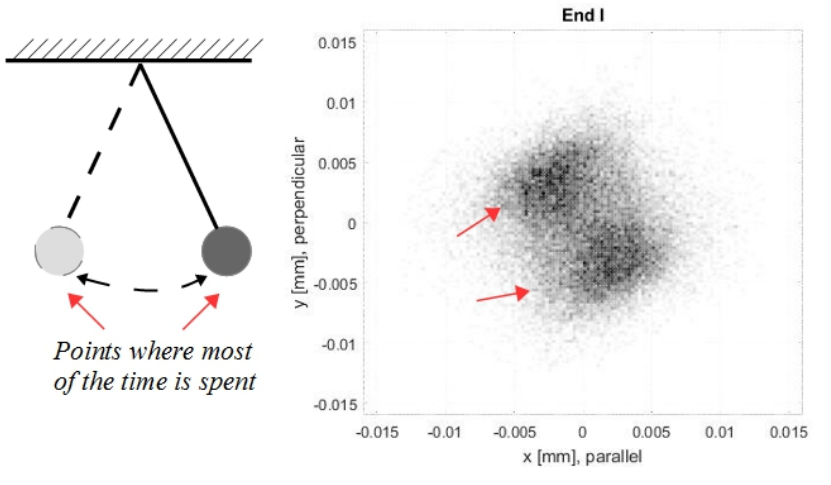

Figure 12. Probability of the TR location if two unstable equilibrium states are observable

a hump in vibrations amplitudes is created. It looks like a typical pattern of the vortex-induced vibrations but considering a small gap between rods and TR location in the 4 th row more investigations are needed to get a definitive conclusion.

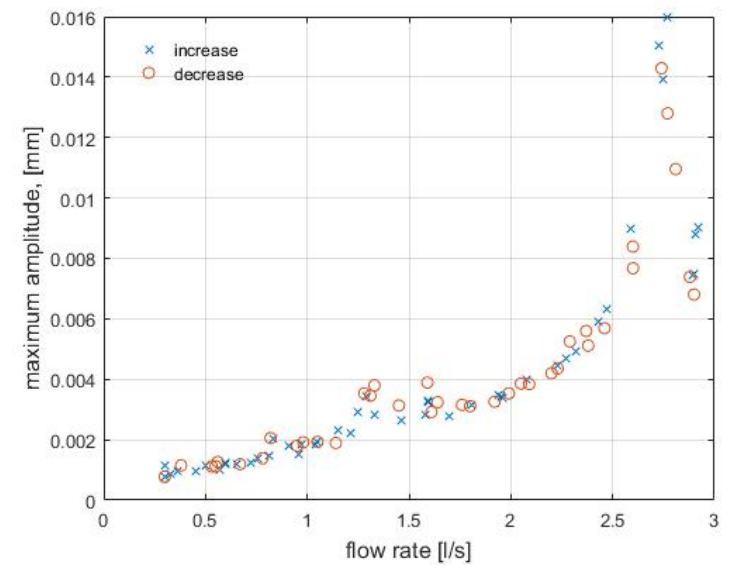

Figure 13. Magnitude of maximum amplitudes of TR depending on flow rates 
Examples of distribution of average displacement amplitudes calculated from accelerometers (Acc1, Acc2 at End I and Acc3, Acc4 at End II) at two flow rates, $Q=$ $2.32 \mathrm{l} / \mathrm{s}$ and $Q=2.74 \mathrm{l} / \mathrm{s}$ is shown in figure 14 . In most cases the random distribution of average amplitudes is observable. Although, at the flow rate $2.74 \mathrm{l} / \mathrm{s}$ in a parallel direction the distribution of amplitudes is similar to the normal distribution (magenta line in Acc2 and Acc4 graph in figure 14). Similar trends are found in other cases when the maximum amplitude of oscillations is reached (between 2.7 and $2.8 \mathrm{l} / \mathrm{s}$ ).
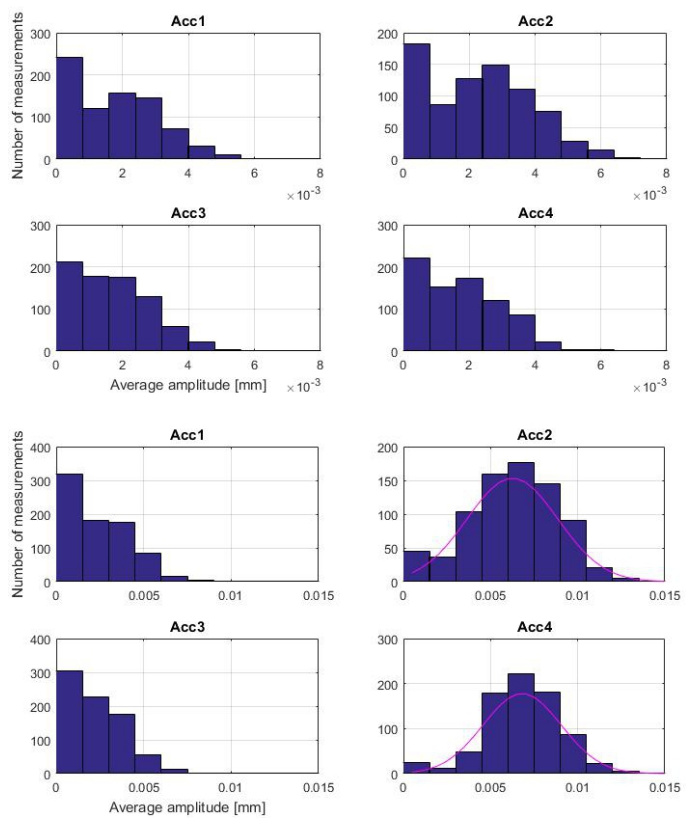

Figure 14. Distribution of average amplitudes of displacements at $Q=2.32 \mathrm{l} / \mathrm{s}$ (top) and $Q=2.74 \mathrm{l} / \mathrm{s}$ (bottom). With magenta line is presented Gaussian distribution function

\section{Conclusions}

A single flexibly-mounted rod is investigated as a model system for the flow-induced vibration evaluation in the closely-packed $(P / d=1.1)$ triangular rod bundle. Characteristic features of the system with relatively small stiffness was analysed. The inlet flow velocity is in a range from $0 \mathrm{~m} / \mathrm{s}$ till $2.06 \mathrm{~m} / \mathrm{s}$ which at maximum gives Reynolds number of 16454 concerning to the rod diameter. Maximum reached oscillation amplitude of the rod is $0.016 \mathrm{~mm}$.
In the analysed system observed two excitation mechanisms. At low flow rates, primary oscillations of the rod come from turbulent fluctuations. When the flow rate reaches a critical value, the rod occurs two unstable equilibrium states and starts large amplitude oscillations. A linear relationship between characteristic frequencies of the system and a flow rate was found at high flow velocity.

The test rod mass impact on the detected frequency and amplitude has been investigated. Correlation between mass increasing and a vibration amplitude increase revealed in the hammer tests. Results show that the fluid added mass effect on the oscillation frequency is minor.

Lagrangian equations for two generalised coordinates: the movement of the test rod midpoint and the rotational angle around the rod centre of inertia, are obtained for perpendicular and parallel direction related to the flow. Comparison of kinetic energies of generalised coordinates shown that in the most cases 2D approximation is allowed.

Based on results of the hammer tests, system parameters for numerical simulations and analytical investigation have been found.

\section{References}

[1] Paidoussis, M.P., Practical experiences with flowinduced vibrations (Springer-Verlag, Berlin, 1980) 181

[2] Andjelić, M., Austermann, R., Popp, K. Journal of Pressure Vessel Technology 114, 336-343 (1992)

[3] Jafari, H.H., Dehkordi, B.G. Journal of Fluids Engineering 135, 031102-1-031102-13 (2013)

[4] El Bouzidi, S., Hassan, M. Journal of Fluids and Structures 57, 264-276 (2015)

[5] Weaver, D.S., Grover, L.K. Journal of Sound and Vibration 59, 263-276 (1978)

[6] Weaver, D.S., Grover, L.K. Journal of Sound and Vibration 59, 277-294 (1978)

[7] Liu, L., Feng, J., Wu, H., Xu, W., Tan, W. Journal of Pressure Vessel Technology 139, 031307-1-031307-8 (2017)

[8] Lin, T., Yu, M. Experimental Thermal and Fluid Science 29, 523-536 (2005)

[9] Weaver, D.S., El-Kashlan, M. Journal of Sound and Vibration 75, 265-273 (1981)

[10] Wheeler, A.J. and Ganji, A.R. Introduction to engineering experimentation, 3rd. ed., (Pearson, 2010) 480

[11] Ziada, S. Journal of the Braz. Soc. of Mech. Sci. \& Eng. 28, 186-199 (2006) 